\title{
Una panorámica de la segregación escolar por nivel socioeconómico en Uruguay
}

\section{An overview of school segregation by socioeconomic level in Uruguay \\ Uma visão geral da segregação escolar por nível socioeconômico no Uruguai}

ISSN 1688-9304 - DOI: https://doi.org/10.18861/cied.2020.11.1.2941

F. Javier Murillo*1

Orcid: https://orcid.org/0000-0002-8003-4133

Raquel Graña Oliver ${ }^{\star * 2}$

Orcid: https://orcid.org/0000-0002-4223-8354

Fecha de recibido: 22/10/2019

Fecha de aprobado: 12/12/2019

\begin{abstract}
Resumen
Este trabajo busca profundizar en el conocimiento de la segregación escolar por nivel socioeconómico en Uruguay, con dos miradas específicas: una a Montevideo y otra a la aportación de las escuelas públicas y privadas. Para ello se realiza una explotación especial de la base de datos del Programa de Evaluación Nacional de Logros Educativos Aristas 2017 desarrollado por el Instituto Nacional de Evaluación Educativa de Uruguay. La muestra, por tanto, está constituida por 15.356 estudiantes de 247 escuelas. En primer lugar, se estima la segregación con los 6 índices más habituales: Disimilitud (ID), Gorard (IG), Raíz Cuadrada (IH), Aislamiento (A), Brecha por Centiles (CGI) e índice Inclusión socioeconómica (IIS). También se estiman los perfiles de segregación y, por último, se analiza la descomposición de la segregación en escuelas públicas y privadas, todo ello tanto para Uruguay en general como para Montevideo en particular. Los resultados apuntan a que Uruguay tiene una segregación escolar por nivel socioeconómico promedio de 0,56 (ID), 0,46 (IG), 0,31 (IA), 0,43 (IH), 0,42 (CGI) y 0,53 (IIS), lo cual puede ser considerado como alto. Asimismo se halló que la segregación presenta valores medio-bajos para los estudiantes de familias con menos nivel socioeconómico y muy alto para los de más nivel. El análisis de la segregación en escuelas públicas y privadas apunta a una muy alta concentración de estudiantes de mayor nivel socioeconómico en las escuelas privadas. No se han detectado diferencias importantes en el estudio de Montevideo. La principal conclusión de este estudio es que Uruguay debe tomar medidas para limitar la segregación escolar de los estudiantes con mayores recursos, asegurando la equidad también en las escuelas privadas.
\end{abstract}

Palabras clave: segregación escolar, nivel socioeconómico, Uruguay, escuelas, educación primaria

\begin{abstract}
This paper seeks to deepen the knowledge of school segregation by socioeconomic level in Uruguay, from two specific perspectives: one as regards Montevideo and the others relating to the contribution of public and private schools. For such purposes, a special exploitation of the National Assessment Program for Educational Achievements Aristas 2017 database developed by the National Institute of Educational Evaluation of Uruguay, has been carried out. The sample, therefore, consists of 15,356 students belonging to 247 schools. First,
\end{abstract}


segregation is estimated through the 6 most common indices: Dissimilarity (ID), Gorard (IG), Square Root (IH), Isolation (A), Centile Gap (CGI), and Socioeconomic Inclusion Index (IIS). Segregation profiles are also evaluated and, finally, the decomposition of segregation in both state and private schools is analyzed. All this, both for Uruguay in general and for Montevideo in particular. The results indicate that Uruguay has an average school segregation by socioeconomic level of 0.56 (ID), 0.46 (IG), 0.31 (IA), 0.43 (IH), 0.42 (CGI) and 0.53 (IIS), which can be considered as high. In addition, it is found that segregation presents medium-low values for students from families with lower socioeconomic status and very high for those belonging to higher socioeconomic levels. The analysis of segregation both in state and private schools points at a very high concentration of students of higher socioeconomic levels in private schools. No relevant differences were detected in the Montevideo study. The main conclusion of this study is that Uruguay must take measures to limit the school segregation of students with greater resources, thus ensuring equity also in private schools.

Keywords: School segregation, Socioeconomic status, Uruguay, School, Basic Education

\section{Resumo}

Esse trabalho procura aprofundar no conhecimento da segregação escolar por nível socioeconômico no Uruguai, com dois olhares específicos: um a Montevidéu e outro à contribuição de escolas públicas e privadas. Para tanto, é realizada uma exploração especial do banco de dados do Programa Nacional de Avaliação de Realizações Educacionais "Aristas 2017", desenvolvido pelo Instituto Nacional de Avaliação Educacional do Uruguai. A amostra, portanto, é composta por 15.356 alunos de 247 escolas. A segregação é estimada mediante 6 índices mais comuns: Dissimilaridade (ID), Gorard (IG), Raiz quadrada (IH), Isolamento (A), Gap central (CGI) e Índice de Inclusão Socioeconômica (IIS). Os perfis de segregação também são estimados e, finalmente, é analisada a discriminação da segregação nas escolas públicas e privadas. Tudo isso, tanto para o Uruguai quanto para Montevidéu. Os resultados sugerem que o Uruguai possui uma segregação escolar por nível socioeconômico médio de 0,56 (ID), 0,46 (IG), 0,31 (IA), 0,43 (IH), 0,42 (CGI) e 0,53 (IIS), que pode ser considerado alto. Além disso, verificou-se que a segregação apresenta valores meio-baixos para estudantes de famílias com menor nível socioeconômico e muito altos para aqueles de nível socioeconômico superior. A análise da segregação nas escolas públicas e privadas aponta para uma concentração muito alta de estudantes de níveis socioeconômicos mais altos nas escolas particulares. Nenhuma diferença importante foi detectada no estudo de Montevidéu. A principal conclusão dessa pesquisa é que o Uruguai deve tomar medidas para limitar a segregação escolar dos alunos com maiores recursos, garantindo a equidade também nas escolas particulares.

Palavras-chave: Segregação escolar, Nível socioeconômico, Uruguai, Escolas, Ensino de primeiro-grau

\section{Introducción}

No hay alternativa: sistemas educativos segregados generan sociedades segregadas y sistemas educativos equitativos, justos e inclusivos generan sociedades cohesionadas y justas. Por diferentes razones, en los últimos años en todo el mundo se está redoblando la preocupación por la segregación escolar y se están tomando medidas, más o menos tímidas, para limitarla en lo posible. Aunque estudios recientes indican que América Latina quizá sea la región con la mayor segregación escolar por nivel socioeconómico del mundo, en esta área este tema aún no se encuentra entre las prioridades políticas y solo el ámbito académico chileno parece estar pendiente del mismo.

Uruguay, por su parte, parece haberse mantenido al margen de esta preocupación. Cierto es que estudios internacionales señalan que es uno de los países de América Latina con menor 
segregación escolar por nivel socioeconómico, lo cual no significa que no se encuentre presente, únicamente significa que en otras regiones del mundo existe una segregación mucho mayor. Hasta ahora no se disponía de estudios que analizaran específicamente la segregación escolar en Uruguay, en gran medida, por la no disponibilidad de datos para ello. Con el desarrollo del estudio evaluativo Aristas 2017 y la liberación de sus bases de datos se está en una posición óptima para comenzar a indagar sobre este tema.

En cuanto a esta indagación Uruguay cuenta con dos características que vale la pena tener en cuenta. En primer lugar, su gran concentración de escuelas en la ciudad de Montevideo, lo que genera que sea importante una mirada específica diferenciada del resto del país. Asimismo, el porcentaje de escuelas privadas es muy bajo en comparación con otros países de la región, lo que la convierte en una enseñanza elitista. Estudiar la aportación de la educación pública y de la privada resulta también de interés.

Con todo ello, en esta investigación se busca profundizar en el conocimiento de la segregación escolar por nivel socioeconómico de Uruguay, con una mirada específica a Montevideo por un lado y a la aportación de las escuelas públicas y privadas por otro. Para ello se llevó a cabo una explotación especial de la base de datos Aristas 2017 de carácter censal.

\section{Marco teórico}

A pesar de que la investigación sobre segregación escolar tiene más de 65 años de existencia (Orfield, Frankenberg y Siegel-Hawley, 2016) y existen miles de trabajos académicos que la han estudiado desde diversas perspectivas, aún no se tiene una definición clara, operativa y consensuada sobre este término. Tampoco existe una única forma de estimar su magnitud ni de interpretar sus resultados.

En esencia, y siguiendo las aportaciones del artículo clásico de Massey y Denton (1989), se puede hablar de dos dimensiones desde las que se conceptualiza la segregación escolar: la dimensión de igualdad o uniformidad (evenness) y la dimensión de exposición (exposure). La primera de ellas define la segregación escolar como la distribución desigual de los estudiantes en las escuelas en función de sus características personales o sociales o por su condición (Dupriez, 2010; Frankel y Volij, 2011; Murillo, 2016, entre otros). La segunda dimensión entiende a la segregación como la probabilidad de que un estudiante se encuentre en la escuela con alguien de su mismo grupo (Allen y Vignoles, 2007; Johnston, Wilson y Burgess, 2004).

Asimismo, no existe un único tipo de segregación escolar. En función de esa característica personal o social de los estudiantes o del grupo del que forma parte podemos hablar de una miríada de segregaciones. Así, si la característica clave es la pertenencia a un grupo étnico/cultural determinado podemos hablar de segregación escolar por origen étnico. Si consideramos el ser nativo o no-nativo, se hablará de la segregación escolar por origen nacional. Si escogemos el nivel socioeconómico de las familias de los estudiantes hablaremos de segregación por nivel socioeconómico (o segregación social), etc. Pero también, en función de las características personales se encuentra la segregación por género, capacidad, rendimiento académico, etc.

Es interesante verificar que el estudio de cada tipo de segregación ha sido predominante según la región del mundo y ha habido una evolución temporal. Así, el inicio de los estudios de la segregación escolar se remonta a Estados Unidos a finales de los años 50 centrados en la segregación étnico-racial. Actualmente sigue habiendo un gran número de 
investigaciones sobre la segregación étnico-racial, antes más centrada en la segregación de afrodescendientes y ahora en la de otras minorías, como las personas procedentes de Latinoamérica (Fiel y Zhang, 2018; Fuller et al., 2019; Santiago, 2019, entre otros). En Europa, el interés ha sido más tardío y se ha focalizado en la segregación de los inmigrantes de primera o segunda generación (Burgess, Wilson y Lupton, 2005; Ivaniushina, Makles, Schneider y Alexandrov, 2019; Karsten, 2010; Simpson, 2007; Teltemann y Schunck, 2016, entre otros) aunque también ha aumentado en los últimos años el interés por la segregación escolar generada por el nivel socioeconómico (Dupriez y Vandenberghe, 2004; Gorard, 2009; Gorard y Hordsoy, 2013; Jenkins, Micklewright y Schnepf, 2008; Murillo y MartínezGarrido, 2017a). En América Latina, con un desarrollo muy reciente, las investigaciones se han centrado esencialmente en este último tipo de segregación (Krüger, 2019; Murillo, 2016; Santos y Elacqua, 2016; Vázquez, 2016).

Esa multiplicidad de tipologías de segregación escolar se ve aún más complejizada por el número de índices de que se dispone para estimar la segregación, aunque aún no existe consenso en torno a su utilización. Si se considera la segregación desde la perspectiva de la uniformidad, los índices más utilizados son el índice de Disimilitud, el índice de Gorard, el índice de Raíz Cuadrada, el índice de Brecha por Centiles, el índice de Información Mutua o el índice Inclusión Socioeconómica (Murillo, 2016). Para medir la segregación desde la dimensión de exposición el índice más habitual es el de Aislamiento.

Las investigaciones sobre segregación escolar en América Latina y el Caribe son escasas y muy recientes. Apenas tienen una década de existencia y se han centrado, en gran medida, en la segregación escolar por nivel socioeconómico. Destacamos cuatro estudios recientes que muestran la magnitud del problema desde una perspectiva internacional y comparada. En primer lugar destacamos el estudio realizado por Vázquez (2016) que analiza la evolución de la segregación escolar por nivel socioeconómico en el mundo desde el año 2000 hasta el 2015 por medio del uso de los datos del Programa Internacional de Evaluación de Alumnos (PISA) desarrollado por la OCDE. Sus resultados son claros: América Latina posee una segregación escolar por nivel socioeconómico relativamente alta en relación a los países de Europa, Asia, América del Norte, Oceanía y África (Vázquez, 2016, p. 113).

En segundo lugar destaca el estudio realizado por Murillo y Martínez-Garrido (2017a) quienes estiman la magnitud de la segregación escolar por nivel socioeconómico, por origen cultural y por origen nacional en América Latina. Emplean los datos derivados del Tercer Estudio Regional Comparativo y Explicativo (TERCE) del Laboratorio Latinoamericano de Evaluación de la Calidad de la Educación. Los autores utilizan el índice de Disimilitud por su gran popularidad en estudios similares, e incorporan como novedad el análisis de dos grupos minoritarios para la estimación de la segregación escolar por nivel socioeconómico: el $25 \%$ de los estudiantes con familias de menor nivel socioeconómico y cultural (Q1) y el $25 \%$ de los estudiantes con familias de mayor nivel socioeconómico y cultural (Q4). Los resultados muestran que el promedio del índice para la segregación escolar por nivel socioeconómico en América Latina es de 0,56, cifra que puede ser considerada casi como hipersegregación. Es mayor la segregación por nivel socioeconómico en el grupo de estudiantes con más recursos con 0,58 puntos, frente a 0,54 de media que posee el grupo más vulnerable. Pese a que estas cifras esconden una importante variabilidad entre países, este dato nos muestra la magnitud del problema al que se enfrenta la región.

En esta línea, el estudio desarrollado por Krüger (2019) utiliza los datos PISA 2015 para analizar la magnitud y las principales características de la segregación escolar por nivel socioeconómico en la región, así como para analizar su impacto en la desigualdad de los 
aprendizajes. En este caso, la autora utiliza tres índices para la estimación de los niveles de segregación: Disimilitud, Información Mutua y Aislamiento. Entre sus resultados se confirman los altos niveles de segregación escolar por nivel socioeconómico en la región dado que, al igual que Murillo y Martínez-Garrido (2017a), se evidencian mayores niveles de segregación entre los estudiantes con familias de mayor nivel socioeconómico.

Por último, otro de los estudios más relevantes sobre la segregación escolar por nivel socioeconómico en América Latina fue publicado en el año 2018 por Murillo, Duk y MartínezGarrido. Esta investigación tiene como objetivo conocer la evolución de la segregación escolar por nivel socioeconómico en las escuelas de América Latina por medio de una explotación secundaria de la base de datos PISA entre los años 2000 y 2015. Los autores estudian tanto la dimensión de uniformidad a través del índice de Gorard como la dimensión de exposición medida a partir del Índice de Aislamiento. Asimismo, con la intención de ofrecer una imagen completa de la segregación escolar por nivel socioeconómico, y siendo conscientes de la importancia de la elección del grupo minoritario (Murillo 2016), se establecen cuatro puntos de corte para conformar los grupos minoritarios: el 10\% y el $25 \%$ de los estudiantes con familias de menor nivel socioeconómico y cultural (P10 y Q1), y el $25 \%$ y el $10 \%$ de los estudiantes con familias de mayor nivel socioeconómico y cultural (Q4 y P90). Pese a la gran diversidad de datos entre países los autores señalan dos hallazgos relevantes:

- La segregación escolar de los estudiantes de familias con mayor nivel socioeconómico es habitualmente mayor que en familias con menor nivel socioeconómico;

- Existe una tendencia al alza en la segregación escolar para los estudiantes de familias con menor nivel socioeconómico y cultural (excepto para Colombia, Costa Rica, Uruguay y Brasil) y una tendencia a la baja para los estudiantes de familias con mayor nivel socioeconómico (excepto para Colombia, Perú y Uruguay).

Existen otras investigaciones interesantes que abordan la magnitud de la segregación escolar por nivel socioeconómico en países determinados tales como Chile (Bellei, 2013; Córdoba, Rojas y Azócar, 2016), Colombia (Duarte, Bos y Moreno, 2012), Ecuador (Murillo y Martínez-Garrido, 2017b), México (Backhoff, 2011; Tapia y Valenti, 2016), Perú (Balarin, 2016; Benavides, León y Etesse, 2014; Carrillo y Murillo, 2019) o Argentina (Jaume, 2013; Krüger, 2018).

Dada la inexistencia de estudios que profundicen en la magnitud de la segregación escolar en Uruguay hemos de recurrir a los estudios internacionales antes señalados para acercarnos a la magnitud del fenómeno en el país.

A nivel de enseñanza primaria se cuenta con la investigación de Murillo (2016) y de Murillo y Martínez-Garrido (2017a). Según estos últimos Uruguay posee un Índice de Disimilitud promedio de 0,52 , lo que ubica al país con un índice de segregación alto. En lo referente a enseñanza secundaria, estimando la segregación escolar por nivel socioeconómico con el Índice de Disimilitud Krüger (2019), para estudiantes de familias con menor nivel socioeconómico la segregación es de 0,40 y de 0,46 para aquellas con mayor nivel. Con respecto al Índice de Aislamiento sus cifras son de 0,37 y 0,50, respectivamente, y con respecto al Índice de Información Mutua es de 0,25. En todo caso, como señala el trabajo de Murillo, Duk y Martínez-Garrido (2018) parece que en los últimos 15 años Uruguay ha reducido la segregación escolar para el P10 y el Q1 y, en cambio, ha aumentado para el Q4 y P90. Krüger (2019), por su parte, usando el Índice de Información Mutua y también con datos de PISA, encuentra que ha habido una ligera disminución en estos años.

Dentro de esta línea de investigación sobre segregación escolar algunos trabajos se han 
centrado en determinar la segregación escolar por nivel socioeconómico en las escuelas públicas y privadas (Arcidiácono et al., 2014; Betts y Fairlie, 2014; Murillo, Belavi y Pinilla, 2018, entre otros). Entre ellos destaca el seminal estudio de Coleman, Hoffer y Kilgore (1982) en el que se abordó el tema de la equidad y la segregación en las escuelas privadas de los Estados Unidos. Sus resultados fueron concluyentes: el nivel socioeconómico de los estudiantes es hasta tres veces más alto en las escuelas privadas que en las públicas lo que genera, asimismo, poco contacto entre estudiantes de diferentes niveles socioeconómicos. Así, hallaron que la proporción de estudiantes con alto nivel socioeconómico cuyos compañeros de clase tienen bajo nivel socioeconómico es de 0,14 en las escuelas públicas y de 0,07 en las privadas.

En América Latina destacan los trabajos de Pereyra (2008), Gasparini, Jaume, Serio y Vázquez (2011), Arcidiácono et al. (2014), Murillo y Martínez-Garrido (2017b) y Krüger (2019). A modo de ejemplo, el estudio de Pereyra (2008) analiza la relación entre el nivel de ingresos de las familias y el tipo de escolarización preferido para sus hijos. Los resultados con datos de 12 países de la región indican que el $9 \%$ de los estudiantes con menores recursos económicos acude a escuelas privadas, frente al $66 \%$ de los estudiantes con mayores recursos. Otro interesante trabajo es el realizado para Argentina por Gasparini et al. (2011). Los autores documentan y analizan la segregación escolar entre los estudiantes de distintos estratos socioeconómicos que asisten a escuelas públicas y privadas desde el año 1986. Muy interesante es el reciente estudio elaborado por Arcidiácono et al. (2014) centrado en el análisis de la evolución de la segregación escolar público-privada por nivel socioeconómico en América Latina. Sus conclusiones apuntan a que, con carácter general, en estas últimas dos décadas ha aumentado esta segregación público-privada en América Latina tanto en educación primaria como en secundaria. Murillo y Martínez-Garrido (2017b), por su parte, analizan la descomposición de la segregación escolar en las escuelas públicas y privadas de los 17 países de América Latina que participaron en el TERCE América Latina y encontraron la fuerte incidencia de la educación privada en la segregación, especialmente para los estudiantes de familias con menor nivel socioeconómico. Krüger (2019), por último, evalúa la descomposición utilizando el Índice de Información Mutua con los datos de PISA 2015 y encuentra que para el conjunto de países de la región la segregación de las escuelas públicas y privadas varía mucho de un país a otro.

En cuanto al Uruguay Murillo y Martínez-Garrido (2017b) encontraron que, para el Q1 -y estimada con el Îndice de Raíz Cuadrada- la segregación de las escuelas públicas es de 0,27 y para las privadas es de 0,68, la segunda más alta de América Latina. Por su parte, para el Q4 la segregación de las escuelas públicas es de 0,25 y de las privadas es de 0,16, la más baja de la región. Krüger (2019), por su parte, encuentra que la segregación de las escuelas privadas genera una segregación mucho más alta que las públicas teniendo en cuanta el poco peso que tienen.

Con esta investigación se busca aportar una panorámica general de la segregación escolar por nivel socioeconómico en Uruguay, con una mirada específica en Montevideo. Concretamente se busca alcanzar los tres objetivos siguientes:

1. Estimar la magnitud de la segregación escolar por nivel socioeconómico en Uruguay y en Montevideo a través de los seis índices de segregación más habituales.

2. Determinar los perfiles de segregación escolar de Uruguay y de Montevideo.

3. Conocer la aportación a la segregación escolar en Uruguay y en Montevideo por parte de escuelas públicas y privadas. 


\section{Método}

Para lograr estos objetivos se ha realizado una explotación especial de la base de datos Aristas 2017, el Programa de Evaluación Nacional de Logros Educativos desarrollado por el Instituto Nacional de Evaluación Educativa de Uruguay. Aristas tiene como objetivo evaluar la calidad del sistema educativo uruguayo desde una perspectiva multidimensional, no reduciendo el análisis a los resultados de las pruebas realizadas a estudiantes sino ampliando el análisis a los contextos sociales y familiares (INEEd, 2018). Participan en el programa estudiantes de $3 .^{\circ}$ y $6 .^{\circ}$ de educación primaria, docentes, directores y familias. Para la selección de la muestra el estudio Aristas realiza un diseño muestral con selección aleatoria sistemática de estratos previamente definidos en relación al tamaño de las escuelas y con sub-estratificación por contexto y categoría de las escuelas. A su vez, es una muestra de selección trietápica donde, en primer lugar, se seleccionan escuelas de manera aleatoria dentro de los estratos; en segundo lugar se seleccionan grupos dentro de las escuelas; $y$, en tercer lugar, se seleccionan todos los estudiantes de los grupos escogidos. En este marco muestral quedan integrados todos los centros educativos públicos y privados de Uruguay con al menos un estudiante de $3 .^{\circ}$ y $6 .^{\circ}$ grado de educación primaria. Finalmente, en Aristas 2017 participaron 247 de las 251 escuelas seleccionadas, por lo que se logró una cobertura de $98,4 \%$.

\section{Cuadro 1. Muestra del estudio}

\begin{tabular}{|l|c|c|c|}
\hline & $\mathbf{N .}^{\circ}$ estudiantes & $\mathbf{N .}^{\circ}$ escuelas & ESCS \\
\hline Uruguay & 7.694 & & \\
3. $^{\circ}$ grado & 7.661 & & $-0,0006$ \\
6. $^{\circ}$ grado & 15.356 & 247 & 0,0025 \\
Total/promedio & & & 0,0010 \\
\hline Montevideo & 2.833 & & \\
3. $^{\circ}$ grado & 2.623 & & 0,1401 \\
6. $^{\circ}$ grado & 5.456 & 171 & 0,1798 \\
Total/promedio &
\end{tabular}

Fuente: Elaboración propia a partir de Aristas 2017.

Como se ha señalado, para aportar una imagen global de la segregación escolar en Uruguay se utilizaron seis índices. En primer lugar, los cuatro índices más habituales: de Disimilitud (D), de Gorard (G), de Raíz Cuadrada (H) y de Aislamiento (A). Los tres primeros miden la dimensión de uniformidad y el último la dimensión de exposición. Matemáticamente se expresan de esta forma:

Índice de Disimilitud

$$
D=\frac{1}{2} \sum_{i=1}^{k}\left|\frac{X_{1 i}}{X_{1}}-\frac{X_{2 i}}{X_{2}}\right|
$$

Índice de Aislamiento

$$
A=\sum_{i=1}^{k} \frac{x_{1 i}}{X_{1}} \frac{x_{1 i}}{T_{i}}
$$

Índice de Gorard

$$
G=\frac{1}{2} \sum_{i=1}^{k}\left|\frac{x_{1 i}}{X_{1}}-\frac{T_{i}}{T}\right|
$$

Índice de Raíz Cuadrada

$$
H=\sum_{i=1}^{k}\left(\left(\frac{x_{1 i}}{X_{1}}\right)-\sqrt{\frac{x_{2 i}}{X_{2}} \frac{x_{1 i}}{X_{1}}}\right)
$$


donde:

- $x_{1 \mathrm{i}}$ representa el número de alumnos del grupo minoritario en la escuela i;

- $x_{2 i}$ representa el número de estudiantes del grupo mayoritario en la escuela i;

- $\mathrm{X}_{1}$ y $\mathrm{X}_{2}$ representan el número total de estudiantes minoritarios y mayoritarios,

- respectivamente, en todas las escuelas de Uruguay (o de Montevideo);

- $\mathrm{T}_{\mathrm{i}}$ representa el número total de alumnos en la escuela i, y

- T representa el número total de alumnos en Uruguay (o en Montevideo).

Para dar la imagen más completa posible se estima la segregación con cuatro grupos minoritarios diferentes: el 10\% de los estudiantes de familias con menor nivel socioeconómico (P10), el 25\% de los estudiantes con familias con menor nivel socioeconómico (Q1), el 25\% de los estudiantes de familias con mayor nivel socioeconómico (Q4) y, por último, el 10\% de los estudiantes de familias con mayor nivel socioeconómico (P90).

Asimismo se estima el Índice de Brecha por Centiles (o GCl, por sus siglas en inglés: Centile Gap Index) cuya fórmula es:

$$
C G I=1-\frac{\mathbf{4}}{N} \sum_{i=1}^{N} \mathbf{l} p_{i}-p_{\text {medi }} \mathbf{I}
$$

donde para cada estudiante i, pi es el percentil de cada estudiante, pmedi es la mediana de los percentiles de la escuela del estudiante i y $\mathrm{N}$ es el número total de estudiantes.

Por último se estima el Índice de Inclusión Socioeconómica (IIS) (Murillo, 2016; OCDE, 2010) expresado matemáticamente de la siguiente forma, a partir del modelo multinivel de dos niveles:

con:

$$
\mathrm{ESCS}_{\mathrm{ij}}=\beta_{0}+\mu_{\mathrm{j}}+\varepsilon_{\mathrm{ij}}
$$

$$
\begin{aligned}
& {\left[\varepsilon_{\mathrm{ij}}\right] \sim \mathrm{N}\left(0, \Omega_{\varepsilon}\right): \Omega_{\varepsilon}=\left[\sigma_{\varepsilon}^{2}\right]} \\
& {\left[\mu_{\mathrm{j}}\right] \sim \mathrm{N}\left(0, \Omega_{\mu}\right): \Omega_{\mu}=\left[\sigma_{\mu}^{2}\right]}
\end{aligned}
$$

De allí se estima el IIS a través del coeficiente de correlación intraclase ( $\rho)$, que sería el IIS:

Para estimar los perfiles de segregación escolar por nivel socioeconómico de Uruguay y de Montevideo se utiliza el Índice de Gorard dado que es el único que no está influido por tamaño del grupo minoritario (Murillo, 2016).

En segundo lugar, para estimar el perfil de segregación de Uruguay y el de Montevideo se usa exclusivamente el Índice de Gorard. La razón es que este índice (frente a otros como el de Disimilitud o el de Aislamiento) no se ve influido por el tamaño relativo del grupo

$$
\rho=\frac{\sigma_{\mu}^{2}}{\sigma_{s}^{2}+\sigma_{\mu}^{2}}
$$


minoritario elegido. Los perfiles se determinan estimando la segregación para un gran número de grupos minoritarios de tamaño creciente. En esta ocasión se estimaron para 19 grupos: el 5\% de los estudiantes de las familias con menos nivel socioeconómico (P5), el 10\% (P10), el 15\% (P15) y así sucesivamente hasta llegar al 50\% (P50), y el 45\% de mayor nivel (P55) hasta el 5\% de las de mayor nivel (P95).

Por último, para determinar la aportación de las escuelas públicas y privadas se usa el Índice de Raíz Cuadrada (H). Esta elección se fundamenta en que es el único de los seis que posee la propiedad de descomposición aditiva, por lo que con él es posible descomponer la segregación total de los subsistemas público y privado (Jenkins et al., 2008; Murillo y Martínez-Garrido, 2007b; Murillo, Belavi y Pinilla, 2018). Dicha formula, ya consignada, es la siguiente:

$$
H=\sum_{i=1}^{k}\left(\left(\frac{x_{1 i}}{X_{1}}\right)-\sqrt{\frac{x_{2 i}}{X_{2}} \frac{x_{1 i}}{X_{1}}}\right)
$$

Es posible dividir el índice en dos partes: la magnitud de la segregación para cada subsistema (intra-subsistema) y la segregación generada entre ambos conjuntamente (inter-subsistemas). El componente $\mathrm{H}$ intra-subsistema es una suma ponderada de la segregación dentro de cada sector g. A su vez el componente $\mathrm{H}$ inter-subsistemas puede expresarse como fracción de H para evaluar qué proporción de la segregación total se debe a la distribución desigual por origen entre un subsistema y otro, en este caso entre escuelas públicas y privadas.

$$
\begin{aligned}
& H=H_{\text {intra }}+H_{\text {inter }} \\
& \text { donde, } \\
& H_{\text {intra }}=\sum_{g=1}^{G} w_{g} H_{g}
\end{aligned}
$$

Con,

$$
w_{g}=\sqrt{\left(\frac{P_{g}}{P}\right)\left(\frac{R_{g}}{R}\right)}
$$

donde, $g=1 \ldots, \mathrm{G}$ subgrupos, y wg es el peso del subgrupo g, Pg y Rg el número de estudiantes en el subgrupo g con respecto al grupo minoritario y mayoritario $P$ y $R$.

Para aportar una mayor información se estimó la descomposición de la segregación en los 19 grupos minoritarios antes considerados, con lo que se obtienen unos perfiles de descomposición nunca antes calculados. 


\section{Resultados}

En correspondencia con los objetivos planteados se presenta la información organizada en tres apartados. En primer lugar se aporta una panorámica general de la segregación escolar por nivel socioeconómico en Uruguay con una mirada específica en Montevideo. En segundo término se profundiza determinando los perfiles de segregación en ambos territorios. Por último se aborda la segregación escolar de los subsistemas educativos públicos y privados.

Para facilitar la lectura se ha optado por incluir en este apartado los gráficos de los perfiles y su descomposición en público y privado (ver los cuadros en anexo).

\section{Panorámica general de la magnitud de la segregación}

Si se atiende a los indicadores estimados es posible afirmar que la segregación escolar por nivel económico en Uruguay es alta. Efectivamente, en promedio, la segregación medida a través del Índice de Disimilitud es de 0,56, en el límite de lo que se considera híper segregación (Massey y Denton, 1989). Ello significa que el 56\% de los estudiantes uruguayos de educación primaria deberían cambiar de escuela para que no hubiera segregación. Cifras análogas arroja la estimación de la segregación escolar con el resto de índices. Así, en promedio, la segregación es de 0,46 según el Índice de Gorard (IG), de 0,31 según el Índice de Raíz Cuadrada $(\mathrm{IH})$ y de 0,42 según el de Aislamiento (IA). El índice de Brecha por Centiles arroja una cifra de 0,42 (CGI) y el de Inclusión Social una cifra de 0,53 (IIS).

Estas cifras promedio ocultan un fenómeno que más adelante se mostrará con toda claridad y que define el sistema educativo uruguayo. La segregación escolar estimada con grupos minoritarios de menores niveles socioeconómicos es claramente inferior que la estimada con los grupos de mayores recursos. Efectivamente, como se ha señalado, para estimar la segregación con los índices de Disimilitud, Gorard, Raíz Cuadrada y Aislamiento (los más habituales) es necesario seleccionar el grupo minoritario. En un primer momento, en esta investigación se han elegido cuatro: el 10\% de los estudiantes de familias con menos nivel socioeconómico y cultural (P10), el 25 de alumnos de familias con menor nivel socioeconómico y cultural (Q1), el 25\% de los estudiantes con mayores recursos (Q4) y el 10\% de los de mayor nivel (P90). Los datos verifican que, para los cuatro índices, la segregación en los grupos minoritarios P10 y Q1 es menor en los grupos minoritarios (cuadro 2).

Cuadro 2. Magnitud de la segregación escolar por nivel socioeconómico en Uruguay

\begin{tabular}{|l|c|c|c|c|c|c|}
\hline & ID & IG & IH & IA & CGI & IIS \\
\hline P10 & 0,4514 & 0,4065 & 0,2121 & 0,2032 & & \\
\hline Q1 & 0,4601 & 0,3452 & 0,2051 & 0,4228 & & \\
\hline Q4 & 0,5980 & 0,4486 & 0,3230 & 0,5832 & & \\
\hline P90 & 0,7204 & 0,6484 & 0,4854 & 0,5160 & & \\
\hline Promedio/Total & $\mathbf{0 , 5 5 7 5}$ & $\mathbf{0 , 4 6 2 2}$ & $\mathbf{0 , 3 0 6 4}$ & $\mathbf{0 , 4 3 1 3}$ & $\mathbf{0 , 4 2 1 7}$ & $\mathbf{0 , 5 3 0 3}$ \\
\hline
\end{tabular}

Nota: ID: índice de Disimilitud; IG: Índice de Gorard; IH: Índice de Raíz Cuadrada; IA: Índice de Aislamiento; CGI: Índice de Brecha por Centiles; IIS: Índice de Inclusión Socioeconómica.

Fuente: Elaboración propia a partir de Aristas 2017. 
Como se ha señalado, junto con la mirada del conjunto de Uruguay se aportan informaciones específicas para Montevideo. La razón es que en esta zona hay una mayor oferta de centros con lo que la segregación está más relacionada con la elección del centro y, con ello, de políticas educativas públicas, y tiene menos relación con el contexto en el que se desarrolla la segregación.

Las estimaciones para Montevideo (cuadro 3) ofrecen cifras muy parecidas a las halladas para el conjunto del país, pero ligeramente más altas en todos los índices y para los 4 grupos minoritarios. Efectivamente, el Índice de Disimilitud promedio es de 0,63 (0,07 más) con lo que es posible hablar de una segregación alta o incluso de híper segregación; el Índice de Gorard es de 0,52 (0,06 más), el de Raíz Cuadrada es de 0,38 (0,07 más), el de Aislamiento es de 0,48 (0,06 más), el de Brecha por Centiles es de 0,44 (0,02 más) y, por último, el de Inclusión Social es de 0,64 (0,11 puntos más).

También se observa una tendencia similar a una mayor segregación para los grupos minoritarios de menor nivel socioeconómico y cultural.

Cuadro 3. Magnitud de la segregación escolar por nivel socioeconómico en Montevideo

\begin{tabular}{|c|c|c|c|c|c|c|}
\hline & ID & IG & IH & IA & CGI & IIS \\
\hline P10 & 0,4818 & 0,4337 & 0,2485 & 0,2177 & & \\
\hline Q1 & 0,5208 & 0,3906 & 0,2596 & 0,4555 & & \\
\hline Q4 & 0,7113 & 0,5336 & 0,4406 & 0,6789 & & \\
\hline P90 & 0,7904 & 0,7114 & 0,5803 & 0,5538 & & \\
\hline Promedio & 0,6261 & 0,5173 & 0,3823 & 0,4765 & 0,4421 & 0,6403 \\
\hline
\end{tabular}

Nota: ID: Índice de Disimilitud; IG: Índice de Gorard; IH: Índice de Raíz Cuadrada; IA: Índice de Aislamiento; CGI: Índice de Brecha por Centiles; IIS: Índice de Inclusión Socioeconómica.

Fuente: Elaboración propia a partir de Aristas 2017.

\section{Perfiles de segregación}

Dado que la magnitud de la segregación depende del grupo minoritario considerado es posible estimar la segregación como una línea más que como un punto, que es la forma en la que tradicionalmente se ha llevado a cabo. Efectivamente, si se estima la segregación para una multitud de grupos minoritarios de tamaño creciente se obtiene una gran cantidad de datos que, representados gráficamente, muestran una línea.

En el gráfico 1 se han representado los perfiles de segregación de Montevideo y de Uruguay. En el cuadro 1A en Anexo figuran los datos completos.

Como puede observarse se verifica con toda nitidez la característica definitoria de Uruguay: la segregación es mucho mayor, y crecientemente mayor cuanto más alto es el nivel socioeconómico y cultural de las familias de los estudiantes, lo que conforma una especie de espiral truncada. Este perfil es radicalmente diferente al de otros países donde el mismo se caracteriza por una forma más simétrica y donde la segregación en los grupos minoritarios más altos es igual a la de los más bajos, es decir, que toma forma de huevo y no de espiral. 


\section{Gráfico 1. Perfiles de segregación escolar por nivel socioeconómico en Uruguay y Montevideo. Índice G para 19 grupos minoritarios}

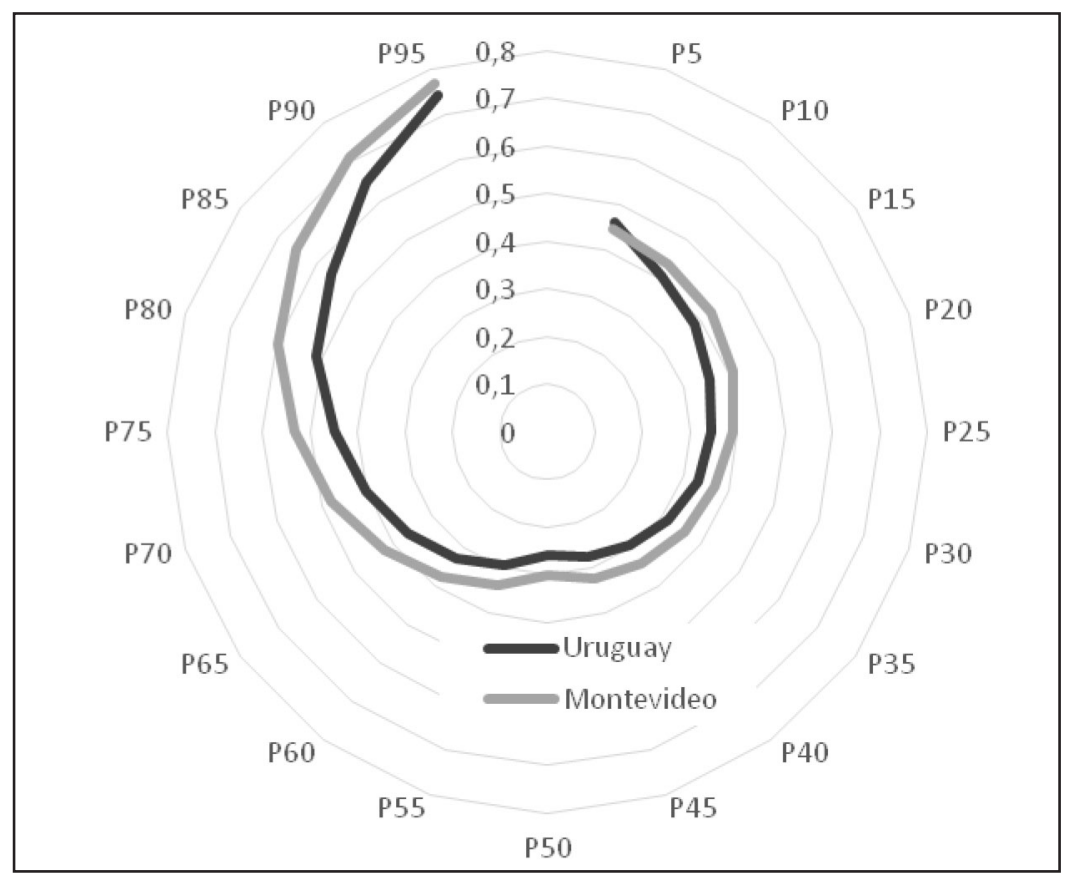

Fuente: Elaboración propia a partir de Aristas 2017.

El perfil de Montevideo es prácticamente el mismo que el del conjunto de Uruguay, pero más alto en prácticamente todas las mediciones. Efectivamente, en promedio es 0,05 más alto, llegando en el P85 a la máxima distancia de 0,09 puntos, y en el P5 es hasta más alta la segregación de Uruguay que la de Montevideo (cuadro 1A).

\section{Segregación en escuelas públicas y privadas}

El perfil de segregación escolar por nivel socioeconómico encontrado tanto para Uruguay como para Montevideo, donde la segregación es baja para los estudiantes de familias de menor nivel socioeconómico y cultural y muy alta para los de mayor nivel, invita a preguntarse por el papel de la educación privada en la configuración de esta situación.

En el cuadro 2A se muestra la descomposición de la segregación del total de las escuelas, la segregación del subsistema de educación pública y de educación privada para Uruguay, y en el cuadro 3A para Montevideo. Una vez más la visión gráfica de estos datos ofrece una imagen muy nítida de lo que acontece. Como puede observarse en el gráfico 2 la segregación de las escuelas privadas es mucho mayor que la de las públicas, al menos hasta el $25 \%$ de los estudiantes con familias de mayor nivel socioeconómico y cultural (P75). A partir de ese punto la mayor segregación se da en las escuelas públicas.

Efectivamente, para el $5 \%$ de los estudiantes de familias con menos nivel socioeconómico (P5) la segregación de las escuelas públicas es de apenas un 0,21, medido a través del Índice de Raíz Cuadrada, mientras que para ese colectivo la segregación de las escuelas privadas llega a un 0,83. Esa mayor segregación se mantiene pero va poco a poco decreciendo hasta llegar, aproximadamente, al 18\% de los estudiantes de familias con mayor nivel socioeconómico, y ambos índices se mantienen casi idénticos en torno a 0,25. Conforme 
el tamaño del grupo minoritario sigue descendiendo, la diferencia vuelve a incrementarse pero en este caso aumentando mucho más en los centros públicos (que para el 5\% de los estudiantes de mayor nivel socioeconómico llega al 0,53), mientras que en los centros privados la segregación se mantiene en los 0,25 para el P95 de los estudiantes.

Si se consideran exclusivamente las escuelas ubicadas en Montevideo, el comportamiento es análogo: una mayor segregación de las escuelas privadas para los grupos minoritarios de menor nivel socioeconómico, y mayor de las escuelas públicas para los grupos de mayor nivel.

\section{Gráfico 2. Segregación escolar por nivel socioeconómico en escuelas públicas y privadas en Uruguay y Montevideo. Índice H para 19 grupos minoritarios.}

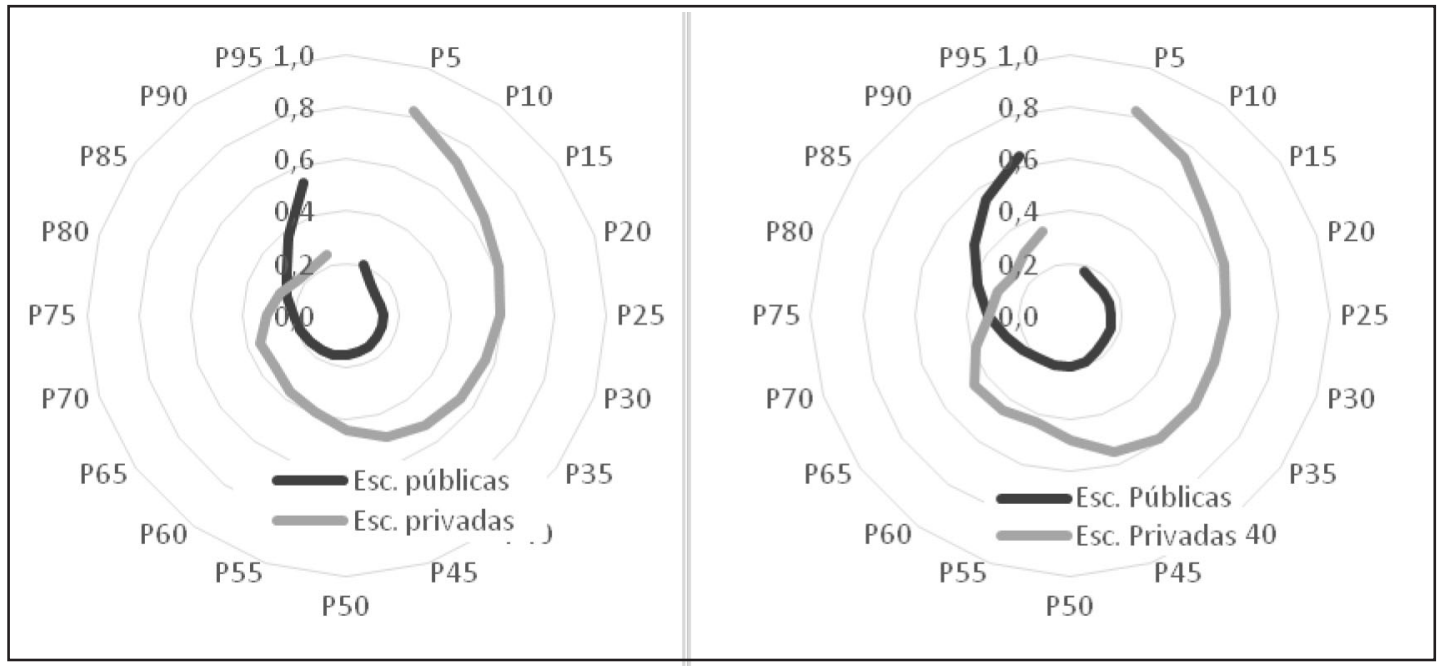

Uruguay

Montevideo

Fuente: Elaboración propia a partir de Aristas 2017.

Una explicación gráfica a estos datos puede obtenerse mostrando a qué tipos de estudiantes escolariza la escuela privada. Como puede observarse en el gráfico 3, el sistema educativo de Uruguay está claramente polarizado. Los estudiantes de niveles socioeconómicos medio y bajo están escolarizados exclusivamente en escuelas públicas, mientras que las clases más altas escolarizan en mayor medida a los estudiantes en escuelas privadas. Esta separación explicaría en parte este especial perfil de la segregación en Uruguay. 


\section{Gráfico 3. Distribución de estudiantes de Uruguay en función de si están matriculados en centros públicos o privados, en cada percentil.}

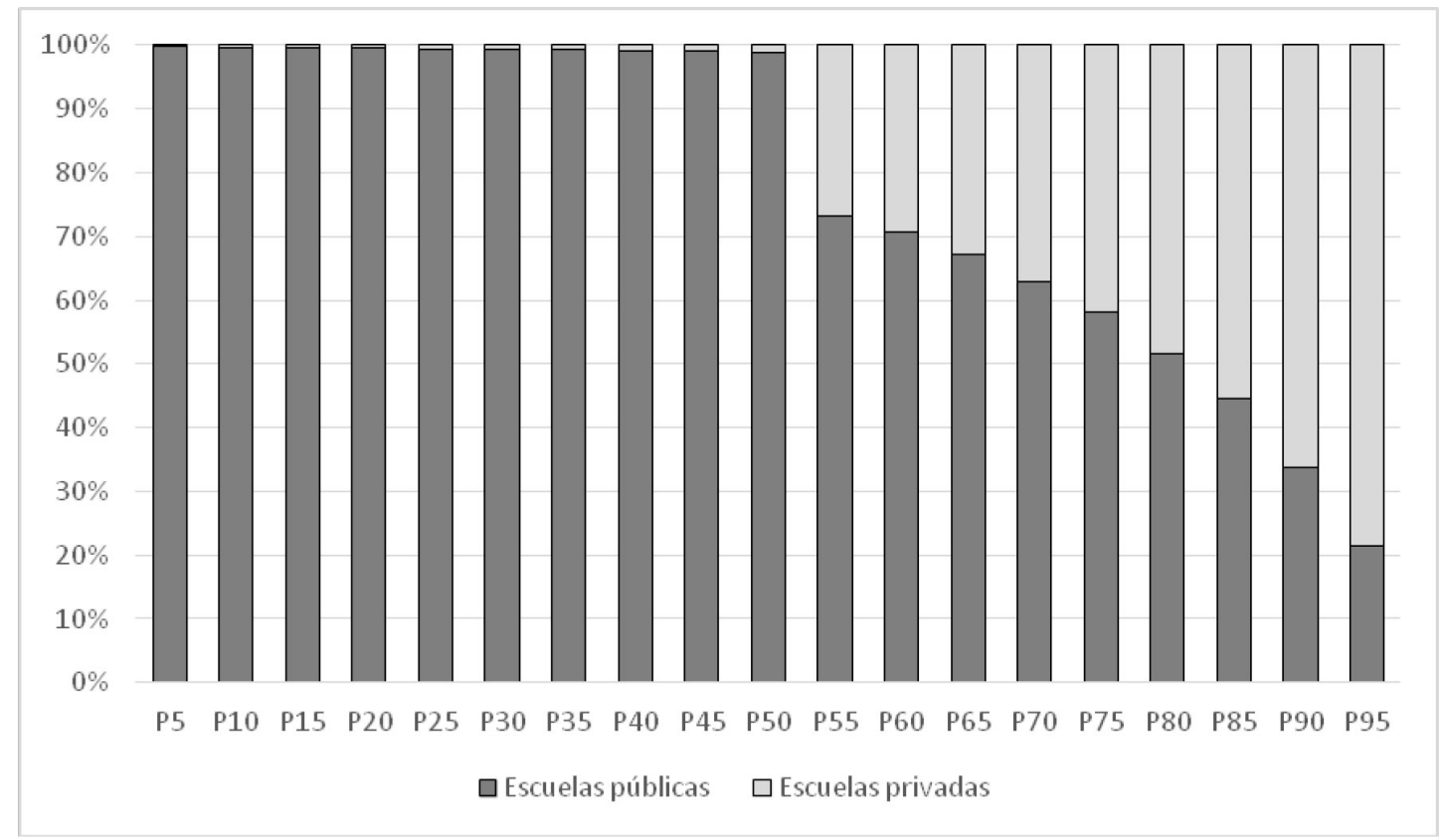

Fuente: Elaboración propia a partir de Aristas 2017.

\section{Discusión y conclusiones}

Esta investigación ha encontrado evidencias empíricas sólidas y consistentes de que la magnitud de la segregación escolar por nivel socioeconómico en Uruguay es en promedio moderadamente alta, aunque una de las más bajas de la región. Sin embargo, un análisis más detallado ha comprobado que presenta una característica diferenciadora frente a otros países. La segregación escolar en Uruguay se caracteriza por una baja segregación para los estudiantes de familias con menos nivel socioeconómico y muy alta para los de mayor nivel. Igualmente, este trabajo ha demostrado que esa segregación para los estudiantes de familias con menos recursos es debida a que los de mayor nivel socioeconómico se escolarizan en gran medida en escuelas privadas de carácter elitista. Con ello puede afirmarse que mientras que la educación pública es globalmente poco segregadora, la educación privada, que solo escolariza a estudiantes de nivel socioeconómico alto o medio alto es responsable de la alta segregación que se produce en esos colectivos. Y todos estos resultados se han encontrado, con pocas diferencias, tanto en el conjunto de Uruguay como en el de Montevideo.

Los datos encontrados en esta investigación confirman lo hallado en las investigaciones internacionales que han estimado la magnitud de la segregación escolar en Uruguay. Efectivamente, Murillo y Martínez-Garrido (2017a) encontraron que, para el conjunto de las escuelas, en base a la utilización de los datos del TERCE y según el Índice de Disimilitud, el índice de segregación escolar por nivel socioeconómico en educación primaria en Uruguay es de 0,49 para el $25 \%$ de los estudiantes con menos nivel socioeconómico y de 0,56 para el $25 \%$ de mayor nivel socioeconómico. Para las escuelas urbanas Murillo y Martínez-Garrido (2017a) encontraron que la segregación en Uruguay es de 0,48 y 0,56. En esta investigación encontramos que, para Montevideo, es de 0,48 y 0,71, respectivamente. Murillo (2016), por su parte, estima no solo el Índice de Disimilitud sino también el de Gorard, el de 
Aislamiento, para P10, Q1 y Q4, y el Índice de Inclusión. Se centra en el hecho de que el índice de Gorard aporta datos de 0,49, 0,37, y 0,42 (para P10, Q1 y Q4, respectivamente), mientras que los autores del presente trabajo han hallado cifras de $0,41,0,35$ y 0,45. Para el índice de Aislamiento Murillo encuentra cifras de 0,26, 0,44 y 0,54 mientras que en este trabajo son de 0,22, 0,46 y 0,68. Para el Índice de Raíz Cuadrada Murillo encuentra cifras de $0,23,0,26$ y 0,44 frente a los del presente trabajo de 0,21, 0,20 y 0,45. Por último, el Índice de Inclusión Socioeconómica hallado por Murillo es de 0,53 y el nuestro es de 0,64. Tal como se ha consignado anteriormente el resto de las investigaciones estiman la segregación en enseñanza secundaria, con lo que la comparación es más forzada.

En resumen, la presente investigación informa acerca de cifras de segregación un poco más bajas para los estudiantes de nivel socioeconómico más bajo, y más altas para los de mayor nivel socioeconómico.

Solo Murillo y Martínez-Garrido (2017b) han abordado la segregación publico-privada a nivel de enseñanza primaria. Como se señaló anteriormente, sus datos indican que para Q1 la segregación bruta de las escuelas públicas es de 0,27 y del 0,68 de las privadas, y para el Q4 de 0,25 y 0,16, respectivamente. Esta investigación encuentra cifras algo diferentes, de 0,15 y 0,68 para el Q1 y de 0,31 para el Q4 tanto para centros públicos como privados; todo ello, como es lógico, con el Índice de Raíz Cuadrada.

Una explicación a estas pequeñas diferencias, además del año de obtención de los datos, es que esta investigación utiliza datos censales (un total de 15.356 estudiantes frente a los datos de los estudios de Murillo, 2016, y Murillo y Martínez-Garrido, 2017a, 2017b) que usan datos muestrales del TERCE, es decir, 5.764 estudiantes. Por tanto, los autores del presente trabajo consideran que los datos aportados en el mismo se ajustan más a la realidad del Uruguay.

En todo caso, los resultados muestran que el sistema educativo uruguayo ha logrado niveles de segregación relativamente bajos para los estudiantes de familias con menos recursos, pero la segregación de los estudiantes de familias de mayor nivel socioeconómico es realmente alta. No debe olvidarse la segregación "por arriba", que también incide en la configuración de una sociedad más justa. La tarea, entonces, ha de enfocarse en el trabajo con esas escuelas privadas elitistas que solo matriculan a estudiantes de familias con recursos.

La imagen que se ha obtenido de la segregación escolar en Uruguay en esta investigación es la más completa hasta ahora, particularmente en el estudio del perfil de segregación, así como de la aportación de la escuela pública y la privada (estatal y no estatal), pero solo da información a nivel de enseñanza primaria y no explicita las razones de la segregación ni las consecuencias, lo que la convierte en un primer paso necesario aunque insuficiente. Para el futuro es necesario seguir indagando en esta realidad por medio de estudios cualitativos que ayuden a comprender esta situación de alta segregación para los niveles más altos así como estudios a nivel de enseñanza secundaria y, desde luego, trabajos longitudinales que aporten información sobre la evolución de esta segregación.

Iniciamos este artículo afirmando que solo se conseguirán sociedades cohesionadas y justas con sistemas educativos inclusivos y justos. Si Uruguay desea avanzar en la construcción de un sistema educativo más justo debe dar pasos urgentes y decididos para reducir esa alta segregación escolar por nivel socioeconómico que evidencia. 


\section{Referencias bibliográficas}

Allen, R. y Vignoles, A. (2007). What should an Index of School Segregation measure? Londres: London School of Economics, Centre for the Economics of Education, CEE.

Arcidiácono, M., Cruces, G., Gasparini, L., Jaume, D., Serio, M. y Vázquez, E. (2014). La segregación escolar público-privada en América Latina. Santiago: CEPAL.

Backhoff, E. (2011). La inequidad educativa en México: diferencias en el aprendizaje de la comprensión lectora en educación básica. Profesorado. Revista de currículum y formación de profesorado, 15(3), 87-102.

Balarin, M. (2016). La privatización por defecto y el surgimiento de las escuelas privadas de bajo costo en el Perú. ¿Cuáles son sus consecuencias? Revista de Sociología de la EducaciónRASE, 9(2), 181-196.

Bellei, C. (2013). El estudio de la segregación socioeconómica y académica de la educación chilena. Estudios Pedagógicos, 39(1), 325-345.

Benavides, M., León, J. y Etesse, M. (2014). Desigualdades educativas y segregación en el sistema educativo peruano. Una mirada comparativa de las pruebas PISA 2000 y 2009. Lima: GRADE.

Betts, J. y Fairlie, R. (2014). Explaining ethnic, racial, and immigrant differences in private school attendance. Journal of Urban Economics, 22(5), 25-46. https://doi.org/10.1006/ juec.2000.2207

Burgess, S., Wilson, D. y Lupton, R. (2005). Parallel lives? Ethnic segregation in schools and neighborhoods. Urban Studies, 42(7), 1027-1056. https://doi.org/10.1080/00420980500120741

Carrillo, S. y Murillo, F. J. (2019). Segregación escolar por nivel socioeconómico en Educación Secundaria en Perú y sus regiones. Revista Peruana de Investigación Educativa, 11.

Coleman, J. S., Hoffer, T. y Kilgore, S. (1982). High school achievement: Public, Catholic, and private schools compared. Nueva York, NY: Basic Books.

Córdoba, C., Rojas, K. y Azócar, J. (2016). Selección de alumnos y co-pago como factores de segregación escolar: Presentación de tres casos de estudio. Psicoperspectivas, 15(1), 102-116

Duarte, J., Bos, M. S. y Moreno, J. M. (2012). Calidad, igualdad y equidad en la educación colombiana. Washington, D.C.: Banco Interamericano de Desarrollo.

Dupriez, V. (2010). Methods of grouping learners at school. París: UNESCO.

Dupriez, V. y Vandenberghe, V. (2004). L'école en Communauté française de Belgique: De quelle inégalité parlons-nous? Les Cahiers de Recherche en Éducation et Formation, 27, 3-26.

Frankel, D. y Volij, O. (2011). Measuring school segregation. Journal of Economic Theory, 146(1), 1-38. https://doi.org/10.1016/j.jet.2010.10.008

Fiel, J. E. y Zhang, Y. (2018). Three dimensions of change in school segregation: A GradePeriod-Cohort Analysis. Demography, 55(1), 33-58. https://doi.org/10.1007/s13524-017$\underline{0632-9}$

Fuller, B. et al. (2019). Worsening School Segregation for Latino Children? Educational Researcher, 1-14. https://doi.org/10.3102/0013189X19860814 
Gasparini, L. C., Jaume, D., Serio, M. y Vázquez, E. (2011). La segregación escolar en Argentina. Reconstruyendo la evidencia. Buenos Aires: CEDLAS.

Gorard, S. (2009). Does the index of segregation matter? The composition of secondary schools in England since 1996. British Educational Research Journal (BERJ), 35(4), 639-652. https://doi.org/10.1080/01411920802642389

Gorard, S. y Hordsoy, R. (2013). Narrowing down the determinants of between-school segregation: An analysis of the intake to all schools in England, 1989-2011. Journal of School Choice, 7(2), 182-195. https://doi.org/10.1080/15582159.2013.791182

INEEd. (2018). Aristas 2017. Informe de resultados de tercero y sexto de educación primaria. Montevideo: Instituto Nacional de Evaluación Educativa.

Ivaniushina, V., Makles, A. M., Schneider, K. y Alexandrov, D. (2019). School segregation in St. Petersburg - the role of socioeconomic status. Education Economics, 27(2), 166-185. https://doi.org/10.1080/09645292.2018.1538408

Jaume, D. (2013). Un estudio sobre el incremento de la segregación escolar en Argentina. Documentos de Trabajo del CEDLAS.

Jenkins, S. P., Micklewright, J. y Schnepf, S. V. (2008). Social segregation in secondary schools: how does England compare with other countries? Oxford Review of Education, 34(1), 21-37. https://doi. org/10.1080/03054980701542039

Johnston, R., Wilson, D. y Burgess, S. (2004). School segregation in multiethnic England. Ethnicities, 4(2), 237-265. https://doi.org/10.1177/1468796804042605

Karsten, S. (2010). School segregation. En OECD, Equal opportunities? The labour market integration on the children of immigrants (pp. 193-209). París: OECD Publishing. https://doi. org/10.1787/9789264086395-en

Krüger, N. (2018). An evaluation of the intensity and impacts of socioeconomic school segregation in Argentina. En X. Bonal y C. Bellei (Eds.), Understanding school segregation: patterns, causes and consequences of spatial inequalities in education (pp. 103-122). Londres: Bloomsbury Academic.

Krüger, N. (2019). La segregación por nivel socioeconómico como dimensión de la exclusión educativa: 15 años de evolución en América Latina. Archivos Analíticos de Políticas Educativas, 27(8).

Massey, D. S. y Denton, N. A. (1989) Hypersegregation in U.S. metropolitan areas: Black and Hispanic segregation along five dimensions. Demography, 26, 373- 391. https://doi. org/10.2307/2061599

Murillo, F. J. (2016). Midiendo la segregación escolar en América Latina. Un análisis metodológico utilizando el TERCE. REICE. Revista iberoamericana sobre calidad, eficacia y cambio en educación, 14(4), 33-60. https://doi.org/10.15366/reice2016.14.4.002

Murillo, F. J. y Martínez-Garrido, C. (2017a). Estimación de la magnitud de la segregación escolar en América Latina. Magis. Revista Internacional de Investigación Educativa, 9(19), 11 30. https://doi.org/10.11144/javeriana.m9-19.emse 
Murillo, F. J. y Martínez-Garrido, C. (2017b). Segregación social en las escuelas públicas y privadas en América Latina. Educação \& Sociedade, 38(140), 727-750. https://doi.org/10.1590/ es0101-73302017167714

Murillo, F. J., Belavi, G. y Pinilla, L. M. (2018). Segregación escolar público-privada en España. Papers. Revista de Sociología, 103(3), 307-337. https://doi.org/10.5565/rev/papers.2392

Murillo, F. J., Duk, C. y Martínez-Garrido, C. (2018). Evolución de la segregación socioeconómica de las escuelas de América Latina. Estudios Pedagógicos, 44(1), 157-179. https://doi.org/10.4067/S0718-07052018000100157

OCDE. (2010). PISA 2009 results: overcoming social background. Equity in learning opportunities and outcomes. París: OCDE.

Orfield, G., Ee, J., Frankenberg, E. y Siegel-Hawley, G. (2016). Brown at 62: School segregation by race, poverty and state. Los Ángeles, CA: Civil Rights Project.

Pereyra, A. (2008). La fragmentación de la oferta educativa en América Latina: la educación pública vs. la educación privada. Perfiles Educativos, 30(120), 132-146.

Santiago, M. (2019). A framework for an interdisciplinary understanding of Mexican American school segregation. Multicultural Education Review, 1-10. https://doi.org/10.108 0/2005615X.2019.1615246

Santos, H. y Elacqua, G. (2016). Socioeconomic school segregation in Chile: parental choice and a theoretical counterfactual analysis. Cepal Review, 119, 123-137.

Simpson, L. (2007). Ghettos of the mind: The empirical behaviour of indices of segregation and diversity. Journal of the Royal Statistical Society, 170(2), 405-424. https://doi.org/10.1111/ j.1467-985x.2007.00465.x

Tapia, L. A. y Valenti, G. (2016). Desigualdad educativa y desigualdad social en México: Nuevas evidencias desde las primarias generales en los estados. Perfiles Educativos, 38(151), 32-54.

Teltemann, J. y Schunck, R. (2016). Education systems, school segregation, and secondgeneration immigrants' educational success: Evidence from a country-fixed effects approach using three waves of PISA. International Journal of Comparative Sociology, 57(6), 401-424. https://doi.org/10.1177/0020715216687348

Vázquez, E. (2016). Segregación escolar por nivel socioeconómico. Midiendo el fenómeno y explorando sus determinantes. Económica, 121-184. 


\section{Anexos}

Cuadro 1A. Perfiles de Segregación escolar por nivel socioeconómico en Uruguay y Montevideo. Índice de Gorard para 19 grupos minoritarios.

\begin{tabular}{|c|c|c|}
\hline & Uruguay & Montevideo \\
\hline P5 & 0,4610 & 0,4475 \\
\hline P10 & 0,4065 & 0,4337 \\
\hline P15 & 0,3825 & 0,4243 \\
\hline P20 & 0,3602 & 0,4089 \\
\hline P25 & 0,3452 & 0,3906 \\
\hline P30 & 0,3324 & 0,3707 \\
\hline P35 & 0,3118 & 0,3565 \\
\hline P45 & 0,2939 & 0,3389 \\
\hline P50 & 0,2751 & 0,3237 \\
\hline P55 & 0,2583 & 0,3011 \\
\hline P60 & 0,2939 & 0,3372 \\
\hline P65 & 0,3263 & 0,3778 \\
\hline P70 & 0,3610 & 0,4232 \\
\hline P75 & 0,4486 & 0,4780 \\
\hline P80 & 0,5101 & 0,5336 \\
\hline P85 & 0,5646 & 0,5962 \\
\hline P90 & 0,6484 & 0,6532 \\
\hline P95 & 0,7427 & 0,7114 \\
\hline Promedio & 0,4066 & 0,7692 \\
\hline P5 & & 0,4566 \\
\hline
\end{tabular}

Fuente: Elaboración propia a partir de Aristas 2017. 
Cuadro 2A. Descomposición de la segregación escolar por nivel socioeconómico en escuelas públicas y privadas en Uruguay. Índice de Raíz Cuadrada (H) para 19 grupos minoritarios.

\begin{tabular}{|c|c|c|c|c|c|c|c|c|}
\hline & \multirow{3}{*}{ Total } & \multicolumn{6}{|c|}{$\mathrm{H}$ intrasistemas } & \multirow{3}{*}{$\begin{array}{c}\mathrm{H} \\
\text { intersistemas }\end{array}$} \\
\hline & & \multicolumn{3}{|c|}{ Escuelas públicas } & \multicolumn{3}{|c|}{ Escuelas privadas } & \\
\hline & & H bruto & Pond. & Aport. & H bruto & Pond. & Aport. & \\
\hline P5 & 0,2582 & 0,2052 & 0,9293 & 0,1907 & 0,8272 & 0,0189 & 0,0157 & 0,0518 \\
\hline P10 & 0,2121 & 0,1551 & 0,9248 & 0,1434 & 0,7247 & 0,0236 & 0,0171 & 0,0517 \\
\hline P15 & 0,1969 & 0,1372 & 0,9201 & 0,1263 & 0,6520 & 0,0267 & 0,0174 & 0,0532 \\
\hline P20 & 0,2006 & 0,1379 & 0,9152 & 0,1262 & 0,6132 & 0,0269 & 0,0165 & 0,0579 \\
\hline P25 & 0,2051 & 0,1398 & 0,9093 & 0,1271 & 0,5920 & 0,0311 & 0,0184 & 0,0596 \\
\hline P30 & 0,2125 & 0,1437 & 0,9029 & 0,1298 & 0,5640 & 0,0331 & 0,0186 & 0,0641 \\
\hline P35 & 0,2166 & 0,1440 & 0,8953 & 0,1289 & 0,5442 & 0,0374 & 0,0203 & 0,0673 \\
\hline P40 & 0,2235 & 0,1474 & 0,8866 & 0,1307 & 0,5183 & 0,0427 & 0,0221 & 0,0707 \\
\hline P45 & 0,2312 & 0,1496 & 0,8765 & 0,1311 & 0,4941 & 0,0463 & 0,0229 & 0,0772 \\
\hline P50 & 0,2390 & 0,1546 & 0,8646 & 0,1336 & 0,4404 & 0,0537 & 0,0237 & 0,0817 \\
\hline P55 & 0,2498 & 0,1616 & 0,8502 & 0,1374 & 0,3908 & 0,0613 & 0,0240 & 0,0885 \\
\hline P60 & 0,2616 & 0,1673 & 0,8332 & 0,1394 & 0,3664 & 0,0703 & 0,0258 & 0,0965 \\
\hline P65 & 0,2756 & 0,1721 & 0,8116 & 0,1397 & 0,3408 & 0,0795 & 0,0271 & 0,1088 \\
\hline P70 & 0,3008 & 0,1859 & 0,7839 & 0,1457 & 0,3479 & 0,0936 & 0,0326 & 0,1225 \\
\hline P75 & 0,3230 & 0,2022 & 0,7503 & 0,1517 & 0,3078 & 0,1132 & 0,0348 & 0,1365 \\
\hline P80 & 0,3648 & 0,2406 & 0,7044 & 0,1695 & 0,2689 & 0,1372 & 0,0369 & 0,1584 \\
\hline P85 & 0,4031 & 0,2824 & 0,6496 & 0,1834 & 0,2330 & 0,1705 & 0,0397 & 0,1799 \\
\hline P90 & 0,4854 & 0,3762 & 0,5607 & 0,2109 & 0,2253 & 0,2127 & 0,0479 & 0,2266 \\
\hline P95 & 0,5901 & 0,5338 & 0,4417 & 0,2358 & 0,2457 & 0,2704 & 0,0664 & 0,2879 \\
\hline
\end{tabular}

Fuente: Elaboración propia a partir de Aristas 2017. 
Cuadro 3A. Descomposición de la segregación escolar por nivel socioeconómico en escuelas públicas y privadas en Montevideo. Índice de Raíz Cuadrada (H) para 19 grupos minoritarios.

\begin{tabular}{|c|c|c|c|c|c|c|c|c|}
\hline & & & & intrasis & temas & & & \\
\hline & Total & & ros esta & & Centr & s no est & tales & $\stackrel{\mathrm{H}}{\mathrm{H}}$ \\
\hline & & H bruto & Pond. & Aport. & H bruto & Pond. & Aport. & \\
\hline P5 & 0,2638 & 0,1744 & 0,8857 & 0,1545 & 0,8218 & 0,0280 & 0,0230 & 0,0863 \\
\hline P10 & 0,2485 & 0,1557 & 0,8778 & 0,1367 & 0,7441 & 0,0405 & 0,0302 & 0,0817 \\
\hline P15 & 0,2516 & 0,1568 & 0,8706 & 0,1365 & 0,6549 & 0,0417 & 0,0273 & 0,0878 \\
\hline P20 & 0,2542 & 0,1565 & 0,8615 & 0,1348 & 0,6212 & 0,0502 & 0,0312 & 0,0882 \\
\hline P25 & 0,2596 & 0,1570 & 0,8519 & 0,1337 & 0,6008 & 0,0558 & 0,0335 & 0,0923 \\
\hline P30 & 0,2664 & 0,1613 & 0,8405 & 0,1355 & 0,5798 & 0,0682 & 0,0395 & 0,0914 \\
\hline P35 & 0,2780 & 0,1642 & 0,8284 & 0,1360 & 0,5907 & 0,0722 & 0,0427 & 0,0994 \\
\hline P40 & 0,2923 & 0,1710 & 0,8142 & 0,1392 & 0,5854 & 0,0790 & 0,0462 & 0,1068 \\
\hline P45 & 0,3116 & 0,1864 & 0,7976 & 0,1487 & 0,5498 & 0,0877 & 0,0482 & 0,1147 \\
\hline P50 & 0,3213 & 0,1953 & 0,7783 & 0,1520 & 0,4791 & 0,1005 & 0,0482 & 0,1212 \\
\hline P55 & 0,3274 & 0,1980 & 0,7560 & 0,1497 & 0,4295 & 0,1162 & 0,0499 & 0,1278 \\
\hline P60 & 0,3472 & 0,2042 & 0,7289 & 0,1488 & 0,4480 & 0,1317 & 0,0590 & 0,1394 \\
\hline P65 & 0,3767 & 0,2247 & 0,6969 & 0,1566 & 0,4539 & 0,1519 & 0,0690 & 0,1511 \\
\hline P70 & 0,4050 & 0,2631 & 0,6596 & 0,1736 & 0,3855 & 0,1772 & 0,0683 & 0,1632 \\
\hline P75 & 0,4406 & 0,3195 & 0,6142 & 0,1963 & 0,3148 & 0,2065 & 0,0650 & 0,1793 \\
\hline P80 & 0,4821 & 0,3732 & 0,5563 & 0,2076 & 0,2963 & 0,2405 & 0,0713 & 0,2032 \\
\hline P85 & 0,5264 & 0,4570 & 0,4927 & 0,2252 & 0,2701 & 0,2823 & 0,0762 & 0,2250 \\
\hline P90 & 0,5803 & 0,5528 & 0,4213 & 0,2329 & 0,2975 & 0,3292 & 0,0979 & 0,2495 \\
\hline P95 & 0,6340 & 0,6447 & 0,3169 & 0,2043 & 0,3375 & 0,3825 & 0,1291 & 0,3006 \\
\hline
\end{tabular}

Fuente: Elaboración propia a partir de Aristas 2017.

* y ** Universidad Autónoma de Madrid (España).

${ }^{1}$ Director de la Cátedra UNESCO en Educación para la Justicia Social y Profesor Titular de Métodos de Investigación y Evaluación Educativa, Universidad Autónoma de Madrid (España). Coordinador, grupo de investigación del Cambio Educativo para la Justicia Social y Secretario Académico, Instituto Universitario de Derechos Humanos, Democracia, Cultura de Paz y No Violencia (DEMOSPAZ), Universidad Autónoma de Madrid. Coordinador General, Red Iberoamericana de Investigación sobre Cambio y Eficacia Escolar (RINACE).

${ }^{2}$ Doctora (Cand.) en Educación sobre segregación escolar y Máster en Calidad y Mejora de la Educación especializada en gestión y liderazgo escolar, Universidad Autónoma de Madrid (España). Educadora Infantil, Universidad de Valladolid (España). Miembro, Cátedra UNESCO en Educación para la Justicia Social y del Grupo de Investigación Cambio Educativo para la Justicia Social, Universidad Autónoma de Madrid. 